treated with the subacetate of lead, which threw down a dense, dirty-white, precipitate. A current of hydro-sulphuric acid was now passed through the mixture, to throw down the lead; the residue gave a pultaceous, dark mass, which, when diluted with water and filtered, became a brown liquid; this latter was boiled in an aqueous solution of white of egg, which, by coagulating, divided the liquid into two parts, one flocenlent, brown, and insoluble, the other liquid and colourless. The latter, being gently evaporated, gave a sirup analogous to the same which had been extracted from the patient's urine. The sirup, being laid on one sile for a few weeks, gave rise to the formation of colourless crystals of a prismatic form, and with a rhomboidal base, in a word, exactly similar to those of sugarcandy. The non-crystallized sirmp having been exposed to a temperature of $26^{\circ}, \mathrm{R}$. with a little beerwashings, the vinous fermentation was soon developed. The quantity of sir up obtained from the pound of blood might have amounted to about one ounce; the crystals of sugar weighed nine grains.

The same chemist has also analysed the blood of a female who died of diabetes mellitus, but he was unable to find the least trace of sugar, either in the blood itself, or in the serum effused into the cavity of the pleura.

\section{VENEREAL DISEASES OF THE TESTICLE.}

Alstract of a Commiznication by J. W. Cusack, M.D., of Stevens's Hospital, Dublin.

So many surgeons of the highest authority have admitted the existence of venereal affections of the testicle, that we must assume the existence of an acute or chronic enlargement of the testis, originating in the action of the venereal poison, to be proved; but here our knowledge, derived from published opinions, ceases. These effections have never been classed, described, or explained, according to their differences, from dissection. From the general conviction of the profession, Mr. Cusack considers himself justified in assuming the existence of a disease of the testis depending on the presence of the venereal poison in the system; at the same time he admits, that as the disease occurs in the more advanced stages of constitutional lues, and after the use of mercury, a doubt must be entertained as to the influence which constitution and treatment may have in producing this disorganization.

The disease, so far as he has observed, commences in the body of the testis, and there is at first little alteration in the form of the organ ; as the enlargement advances, the tumour becomes more globular, the epididymis soon being lost in the general mass ; the tumour has a fleshy feel, but differs much in density in different parts. Partial adhesions in the cavity of the tunica vaginalis, combined with effusions into that cavity, even independent of the internal charges which may be going on, render plimary smoothness and uniformity of the surface an uncertain symptom; so uncertain indeed and so little uniform are the primary appearances, that Mr. Cusack believes that the best practitioners would be unable to make a perfect diagnosis of the disease, if unacquainted with the history of the case, and the attending circumstances. Its termination is either resolution or suppuration or induration, and the formation of granular bodies, ending in total destruction of the functions of the organ. Mr. Cusack hesitates to believe, and is unable to affirm from his own practice, that this affection of the testis is met with in the earlier stages of secondary symptoms, or during the presence of any of the forms of true papular eruption. The acute form accompanies venereal hectic, pains in the bones, and either a scaly eruption or perhaps a solitary spot, apparently belonging rather to the genus acne; but these instances are comparatively rare, and of fifty patients at present under treatment in Stevens's Hospital, there is not one case to adduce in confirmation of such an opinion. The patients who suffer from this affection are those persons who labour under affections of the periosteum and bones, and bear the marks of having suffered from pustular and tubercular eruptions. Specimens of the disease were subnitted to the Surgical Society, and these were wholly de. rived from persons whose constitutions were broken down from the protracted forms of the disease with which practitioners are familiar, but neither from his own opportunities nor from any other source could $\mathrm{Mr}$. Cusack exhibit a specimen of the changes which take place in the more curable forms of secondary syphilis. Ten preparations were laid on the table, exhibiting the disease in all the stages of its progress, from a small circumscribed tubercle, in an otherwise sound testis, to the contracted, indurated, and completely disorganized gland. The structure of the tubercle is rather soft, but harder than common scrofulous tumour, and surrounded by a thickened layer resembling a cyst, the product of inflammatory action. In one preparation the tubercle was in the lower part of the testis, which was otherwise so sound that the epididymis admitted of injection by mercury, while in the opposite testis the tubercle was softened, and contained a glairy fluid.-Dub. Sourn., Nov. 1835. 


\section{OPERATIONS FOR STONE.}

The following statistique of operations performed at the Hospital of Incurables and of St. Mary, at Naples, during the Spring of 1835, we extract from the Gaz. Med. de Paris, No. 44 :--

The neck of the bladder was divided downwards and to one side. Professor De Revzr is in the habit of publishing annually the statistics of all the operations for stone which are publicly performed at Naples How much would be gained by science if the surgeons of our great hospitals were compelled to imitate this example, for the principal operations which they are called upon to perform! We should then have a body of authentic facts upon which confidence might be placed, and from which deductions of the highest value might be drawn.

At the Hospital of Incurables, ten operations were performed; six of the patients were below ten years of age; the others had not reached that of puberty. In four cases the stone was large; in three it was small; in three of a moderate size. There were nine males and one female. Only one patient died of the ten; after having cut into the bladder, the surgeon was unable to extract the stone. The bladder, after death, was found much inflamed, almost gangrenous, and the mucous membrane was reduced to a kind of pulp.

At the Hospital of St. Mary, four patients were cut, and all recovered: three were below fifteen years of age.

Since the year 1821, up to the present time, 454 patients have been operated upon in these two hospitals. 439 males, 15 females; or 216 children, 191 adults, and 47 old. Of these there were cured 388 ; died 66 ; giving an average of one death in nearly every seven cases.

\section{ARtifictal ANUS CURED BY A NEW process.}

Is the month of September last, M. AMus . SAT, of Paris, was called on to see a child two days old, who had not yet passed any meconium. The anus existed, and was well formed, but the rectum terminated by an opening in the vagina. On examination it was found that the rectum did not exist for two inches from the anus. M. Amussat, therefore, determined on seeking the extremity of the great intestine, separating it from its attachment, and fixing it by suture to the opening in the skin; for this purpose he made a $I$ incision between the vaginal opening and the ants, and having introduced the finger by this opening, he separated the vagina from the coccyx and sacrum, until he arrived at the blind end of the great in. testine, which was placed above, to the right side of the sacro-vertebral angle. Arrived at this point, the manouvres produced a desire to evacuate the meconium. The gut was recognised by Messrs. Deneux and Lebaudy, who were in attendance. It was seized with a hook, separated from the surrounding parts by the finger, and drawn gently down to the opening in the skin, where it was fixed with several points of suture. It is now twenty-eight dayrs (said M. Amussit at a late meeting of the Academy of Medicine) since the operation just described has been performed, and no accident has occurred, the child being in the most satisfactory state.

\section{INQUEST AT FARNHAM.}

\section{To the Editor of THE LANCET.}

SrR,- The details of the subjoined case will go farther to prove the necessity for the appointment of medical coroners, than any which I have yet known to be brought before the public. The verdict delivered was "Accidental Poisoning," and nux vomica was the poison sworn to have caused the fatal result. The medical evidence and a general outline of the inquest $I$ transmit for publication, well assured that the pages of The LaxceT will be open to the insertion of facts of so much general importance, especially as the character of a respectable individual is involved in the inquiry, the medical evidence having guided the coroner and jury in the delivery of the charge and the verdict. No other channel offers to clear the person on whom odium is attempted to be cast (the local opportunity being hermetically sealed), and he here, therefore, begs to challenge those opinions which can decide the question at issue. The details are given from memory, for on applying to the coroner for a copy of the depositions, he declined granting one, on the ground of the concession being illegal, though he politely read the depositions to me; and as $\mathrm{Mr}$. Portello appealed to $\mathrm{me}$ in his distress, I am bound to carry the inquiry forward to an issue, that I may not be involved by undertaking a defenceless cause.

On Thursday, the 8th of Octoler, a travelling man and woman called at the shop of Mr. Postello, a druggist in Farnham, and requested some medicine for a sick child of thirteen months old. The child was drowsy, and its bowels were much confined, to ob- 